\title{
archOnline@JCU
}

This is the Accepted Version of a paper published in the journal Global Ecology and Biogeography:

Siqueira, Alexandre C., Oliveira-Santos, Luiz Gustavo R., Cowman,

Peter F., and Floeter, Sergio (2016) Evolutionary processes

underlying latitudinal differences in reef fish biodiversity. Global

Ecology and Biogeography, 25 (12). pp. 1466-1476.

http://dx.doi.org/10.1111/geb.12506 


\section{Evolutionary processes underlying reef fish latitudinal differences in biodiversity}

2

3 Alexandre C. Siqueira ${ }^{\mathrm{a} *}$; Luiz Gustavo R. Oliveira-Santos ${ }^{\mathrm{b}}$; Peter F. Cowman ${ }^{\mathrm{c}, \text {; }}$ Sergio R.

$4 \quad$ Floeter $^{\mathrm{e}}$

5

6

${ }^{\text {a }}$ Departamento de Ecologia e Zoologia, Universidade Federal de Santa Catarina, Florianópolis, SC, 88040-970, 7

Brazil. Email: alesixbio@gmail.com

8

${ }^{\mathrm{b}}$ Departamento de Ecologia, Universidade Federal do Mato Grosso do Sul, Campo Grande, MS, 79070-900,

9 Brazil. Email: gu_tapirus@hotmail.com

${ }^{\mathrm{c}}$ Department of Ecology and Evolutionary Biology, Yale University, New Haven, CT, 06511, USA.

${ }^{\mathrm{d}}$ Centre of Excellence for Coral Reef Studies, James Cook University, Townsville, QLD, 4810, Australia. Email: peter.cowman@jcu.edu.au

${ }^{\mathrm{e}}$ Departamento de Ecologia e Zoologia, Universidade Federal de Santa Catarina, Florianópolis, SC, 88040-970,

Brazil. Email: sergio.floeter@ufsc.br

\section{*Corresponding author:}

Alexandre C. Siqueira

Marine Macroecology and Biogeography Lab, Departamento de Ecologia e Zoologia, Universidade

Federal de Santa Catarina, Florianópolis, SC, 88040-970, Brazil. Email: alesixbio@gmail.com

Phone: +5548 3721-5521 / Fax: +55 48 3721-5156

Running-title: Evolution and reef fish latitudinal biodiversity

Keywords: diversification, speciation, extinction, dispersal, species richness, models of evolution

Number of words in the Abstract: 245

Number of words in main body: 4931

Number of references: 48 


\section{ABSTRACT}

Aim: To examine the dynamics among processes of speciation, extinction and dispersal in marine environments using phylogenies to reveal the evolutionary mechanisms that promote latitudinal differences in biodiversity. Using phylogenetic comparative methods we assess whether tropical reef fish lineages show higher diversification rates and whether the majority of extratropical reef fish lineages have originated from tropical areas.

Location: Shallow water tropical and extratropical reefs globally.

Methods: Using fossil calibrated phylogenies for four reef associated fish families (Chaetodontidae, Labridae, Pomacentridae and Sparidae) we apply evolutionary models (GeoSSE and HiSSE) that allow the estimation of speciation, extinction and dispersal rates associated with geographic ranges and exploring potential biases from unsampled characters.

Results: We found that tropical lineages show higher rates of speciation and tended to have lower extinction rates. Overall, we identify higher net diversification rates for tropical lineages when compared to those in extratropical regions in all four families. Rates of dispersal tended to be higher for lineages with tropical origins expanding into extratropical regions. Within the family Labridae, two tropical lineages were found to exhibit higher net diversification rates, above that expected by latitudinal differences.

Main conclusions: Our results offer support for the predictions of the 'out of the tropics' and 'evolutionary speed' models of evolution, both of which highlight the marine tropics as an important evolutionary engine promoting latitudinal differences in reef fish biodiversity. Moreover, we find that two tropical labrid lineages are undergoing exceptional diversification associated with additional traits, possibly linked with extreme sexual dichromatism observed in both clades. 


\section{INTRODUCTION}

Although being the most widespread macroecological pattern in the world, the latitudinal diversity gradient (LDG) remains poorly understood with respect to its generating processes (Hillebrand, 2004). This limited understanding stems from a lack of focus on the historical and evolutionary factors driving this pervasive biodiversity pattern. The uneven distribution of tropical and extratropical lineages in phylogenetic trees is an evolutionary property that arises from the LDG depending on the dynamics between speciation and extinction (Mittelbach et al., 2007). Besides this, the phylogenetic component of the LDG depends on the process of lineage dispersal between different ecological zones (Ricklefs, 2006). Thus, every evolutionary hypothesis concerning the LDG must take into account at least one of these three fundamental processes that alter species richness: speciation, extinction and dispersal (Dowle et al., 2013). Considering these processes, there are four main hypotheses to explain the phylogenetic properties arising from the LDG. (1) The 'evolutionary time' hypothesis (Fischer, 1960), considers that tropical lineages have had more time to diversify because tropical environments remained more stable throughout geological time. According to this hypothesis, evolutionary rates would be equal among geographic regions, however, tropical lineages would be older than extratropical ones, leading to more extant species in the tropics. (2) The 'tropical niche conservatism' hypothesis (Wiens \& Donoghue, 2004), assumes that species that share common ancestry tend to share niche affinities. It proposes that most lineages would have originated in the tropics because tropical areas had greater geographical extent in recent geological past and that the transition to extratropical zones would be rare. In terms of evolutionary rates, speciation and extinction were supposed to be equal among geographic regions and dispersal would be more frequent from extratropical zones to the tropics. (3) The 'out of the tropics' (Jablonski et $a l ., 2006)$ is an explicit hypothesis about differences in all evolutionary rates. It suggests that 
lineage origination is higher in the tropics, extinction rates are higher or equal in extratropics compared to the tropics, and that tropical lineages disperse to the extratropics more frequently than the reverse. (4) Finally, the 'evolutionary speed' hypothesis (Rohde, 1992) proposes that speciation rates would be higher in tropical environments, mainly due to increased rates of molecular evolution in the tropics (reviewed in Dowle et al., 2013). Thus, this hypothesis considers that extinction and dispersal rates would not vary among geographic regions.

Recent studies are helping to disentangle these evolutionary processes by using timecalibrated phylogenies to make explicit tests of speciation, extinction and dispersal rates between tropical and extratropical lineages (Pyron \& Wiens, 2013; Pyron, 2014; Rolland et al., 2014). Their main results suggest higher speciation rates for tropical lineages in amphibians and mammals (Pyron \& Wiens, 2013; Rolland et al., 2014), but not in squamate reptiles (Pyron, 2014), while higher extinction rates have been reported for extratropical lineages in all groups. As for dispersal rates, these studies showed more lineage dispersal from the extratropics to the tropics in amphibians and squamate reptiles, while mammals follow the opposite pattern. These results reinforce the idiosyncratic nature of evolutionary processes among taxonomical groups, yet they have focused on terrestrial organisms. It is still unclear which processes have been important in marine systems for generating latitudinal patterns of biodiversity.

Although many marine groups exhibit a strong LDG (Hillebrand, 2004), there remains a gap in our knowledge of the underlying phylogenetic component, especially in diverse systems like coral reefs. These environments tend to occupy tropical latitudinal bands, but peripheral extratropical conditions may also permit the establishment of complex, non-coral dominated reef communities (Ebeling \& Hixon, 1991; Fig. 1). It is the transition from tropical coral dominated reef to extratropical rocky reef and kelp forest systems that forms the latitudinal diversity gradient for reef-associated organisms. Coral reef environments are recognized as 
important promoters of lineage diversification, in particular for associated fishes, where they have acted as refugia for biodiversity in periods of elevated extinction (Cowman \& Bellwood, 2011; Pellissier et al., 2014). Reef fishes not only exhibit a marked latitudinal gradient with more species in the tropics but also a striking longitudinal gradient with species richness peaking in the Indo-Australian Archipelago (Mora et al., 2003). Many efforts have been devoted to understand the contemporary factors that influences these patterns (e.g. Bellwood et al., 2005; Tittensor et al., 2010), with geometric (reef area and costal length) and biogeographic variables identified as powerful predictors for reef fish species richness (Parravicini et al., 2013). However, as yet, no study has attempted to quantify the roles played by processes of speciation, extinction and dispersal in large-scale patterns of reef fish richness among tropical and extratropical regions.

Here, we employed phylogenetic comparative methods to test predictions of the evolutionary hypotheses concerning reef fish latitudinal differences in biodiversity. We used time-calibrated phylogenies of four families that are known to have representatives in both tropical and extratropical reefs to test for differences in speciation, extinction and dispersal rates among lineages. Although these three processes have not been tested for reef fishes in the context of the LDG, they are recognized as important drivers of contemporary species richness patterns for this system (Mora et al., 2003). Specifically, we assessed which evolutionary hypothesis ('evolutionary time', 'tropical niche conservatism', 'out of the topics' or 'evolutionary speed') drives the formation and maintenance of higher reef fish diversity in the tropics.

\section{METHODS}

\section{Reef fish evolutionary relationships}


We examined four percomorph families with high phylogenetic resolution, known to be associated with both tropical and extratropical reef environments (Bellwood \& Wainwright, 2002): Chaetodontidae, Labridae, Pomacentridae and Sparidae. The Chaetodontidae, Labridae and Pomacentridae are among the most species rich families in reef environments and have a good phylogenetic resolution. However, they are predominantly associated with tropical coral reefs. To capture a good representativeness both in terms of species richness and variation in occupation of tropical vs. extratropical environments we also included the family Sparidae, which is characteristic of extratropical reefs (Bellwood \& Wainwright, 2002). For our phylogenetic comparative methods, we used recent published chronograms for each family.

The Chaetodontidae phylogeny was reconstructed with Bayesian inferences using four mitochondrial genes and four nuclear genes (see Cowman \& Bellwood, 2011). It was calibrated using fossil data and included 96 species from all nominal genera for the family.

The labrid phylogeny from Cowman \& Bellwood (2011) was combined with the parrotfish phylogeny of Choat et al. (2012). This was accomplished by grafting the parrotfish clade into the labrid tree at the appropriate node using the 'ape' package (Paradis et al., 2004) in R (R Core Team, 2014). This larger phylogeny included 303 species from 70 genera.

For the Pomacentridae we employed the chronogram of Frédérich et al. (2013). This represents the most recent molecular and phylogenetic analysis of the family with Bayesian inferences using three nuclear and four mitochondrial genes. This chronogram also used fossil data and comprised 206 species from 28 of 29 recognized genera for the family.

For the Sparidae, we used the most well sampled phylogeny to date (Santini et al., 2014). This fossil calibrated phylogeny was built with three mitochondrial and two nuclear genes, and included 91 species with representatives from all recognized genera for the family (see Santini et al., 2014). 


\section{Geographic data}

We assessed the geographic ranges of all nominal species in each family through four different sources: published data in books and papers (e.g. Allen, 1991; Randall, 2005; Kuiter, 2010); Catalog of Fishes (Eschmeyer, 2014); IUCN's red list (IUCN, 2014); and FishBase (Froese \& Pauly, 2014). The range data for each species was cross-checked between these four sources to avoid any probable large-scale geographic error. We then categorized each species according to the presence or absence in tropical and extratropical regions, leading to three discrete geographical states: tropical, extratropical and widespread. This categorization was made considering the isocryme of $20^{\circ} \mathrm{C}$ (Fig. 1) - mean sea surface temperature for the coldest month - as a latitudinal distribution limit for tropical marine fauna (Briggs, 1974). We also calculated the proportion of species in each of the three geographical states that were present in each family's phylogeny (Table 1).

\section{Phylogenetic Comparative methods}

To test for differences in speciation, extinction and dispersal rates between tropical and extratropical regions, we used the model of "Geographic State Speciation and Extinction" (GeoSSE; Goldberg et al., 2011), implemented in the R package 'diversitree' (FitzJohn, 2012). This is a phylogenetic comparative method that uses a similar mathematical formulation as the BiSSE ("Binary State Speciation and Extinction”) model, but enables tests of evolutionary rates associated with geographical states rather than with discrete character states. Recent concerns have been raised about the BiSSE and related methods (Rabosky \& Goldberg, 2015), regarding the possibility of phylogenetic pseudoreplication and high Type I error rates associated with speciation estimates. To mitigate these potential biases we employed three approaches: (1) We 
replicate the GeoSSE models across families of varying size and tip state ratio (Table 1). (2)

We built models using the "Hidden State Speciation and Extinction" (HiSSE) framework (Beaulieu \& O'Meara, 2016), a new approach which considers that unmeasured characters ('hidden traits') related to the ones in the model might be influencing diversification estimates. (3) We run simulation models to explicitly access the Type I error rates associated with GeoSSE model in our data. By assessing models across multiple reef fish families we can identify robust patterns, and with the implementation of HiSSE we can explore the potential effects of unsampled character traits to deal with the problem of phylogenetic pseudoreplication. Simulation tests help to assure that our results are not examples of mistaken inferences by the models (Rabosky \& Goldberg, 2015).

The GeoSSE model included three speciation parameters: $\mathbf{s}_{\text {Trop }}$ and $\mathbf{s}_{\mathrm{Ex}}$ representing the divergence of an 'endemic' (restricted to a tropical or an extratropical range) ancestral species producing two daughters in the same geographical state, or a widespread lineage giving rise to tropical or extratropical endemic lineages; and $\mathbf{s}_{\mathrm{Btw}}$ representing the divergence of a widespread lineage between regions producing two daughter lineages, one in each geographical state. The parameters included in the model associated with extinction are: $\mathbf{x}_{\text {Trop }}$ and $\mathbf{x}_{\mathrm{Ex}}$ representing global lineage extinction or range contraction of a widespread lineage. The model also includes the dispersal parameter $\mathbf{d}_{\text {Trop }}$ representing range expansion of tropical lineages and $\mathbf{d}_{\mathrm{Ex}}$ representing range expansion of extratropical lineages. During model construction, all these parameters can be constrained or allowed to vary freely between regions.

We used the four chronograms to test several models that were built according to the predictions of each evolutionary hypothesis proposed to explain the LDG. First, we built unconstrained (full) models in which the seven parameters were allowed to vary freely, representing the notation of the 'out of the tropics' model of evolution that predicts differences 
in all rates. We also built models by constraining all rates to be equal among regions, representing the 'evolutionary time' hypothesis that predicts no difference in evolutionary rates. To represent the 'evolutionary speed' hypothesis, we built models in which only speciation rates varied geographically, constraining extinction and dispersal rates to be equal among regions. Finally, we constrained speciation and extinction to fit the 'niche conservatism', that predicts higher dispersal rates from the extratropics to the tropical regions. As it is not possible to constrain the direction of parameter values when fitting our models, consistency with an evolutionary hypothesis depends on a superior fit and finding the correct parameter direction. From the four evolutionary models, we built four other sub-models by constraining the $\mathbf{s}_{\mathrm{Btw}}$ parameter to be equal to zero to assess the effect of between-region speciation mode in each family. These combinations resulted in a set of eight models varying from the fully unconstrained ('out of the tropics') model with seven parameters to the totally constrained ('evolutionary time') model with only three parameters (Table S1 - Appendix S1 in Supporting Information). parameter space with 50 different combinations of initial parameters to assure we reached the maximum likelihood (global optimum). After that, we used one of these convergent results as starting values to run our models. We then compared our set of models using Akaike Information Criterion (AIC), choosing the best-fit model by the lowest delta AIC score $(\triangle \mathrm{AIC}=0)$. To assess the power in model selection for our original data, we also used a Monte Carlo approach described by Boettiger et al. (2012). We simulated 100 phylogenies with parameters estimated under GeoSSE for each model ('out of the tropics', 'evolutionary speed', 'evolutionary time' and 'niche conservatism'), which resulted in four sets of 100 simulated 
set of simulated phylogenies by re-estimating and comparing model fit for the most complex model ('out of the tropics') against each respective simpler models ('evolutionary speed', 'evolutionary time' and 'niche conservatism').

After model selection, we estimated the parameters for the best-fit model in each family and sampled their posterior probability distributions using Markov chain Monte Carlo (MCMC) to account for estimate uncertainty. The MCMC was run for 1000 generations using exponential priors from the initial likelihood function. With the resulting samples from MCMC runs we calculated the net diversification rates of each family by subtracting extinction rates from speciation rates. Within the GeoSSE model it is possible to account for incomplete sampling by including the percentage of species from each geographical state that are present in the trees. We performed the modeling procedure as described above including the percentage of sampling species for each family's phylogeny (Table 1). For a better visualization of changes through time in geographical states, we also performed a simple marginal ancestral state reconstruction with GeoSSE's initial likelihood function (Figs. S1-S4 - Appendix S2 in Supporting Information).

We compared the regular time-constant GeoSSE model to a recently modified version of this model that allows the incorporation of time dependency in evolutionary rates with a function developed by Rolland et al. (2014). This function was created to avoid a potential bias of time variance in the estimation of evolutionary rates. We implemented the time-variable model as in Rolland et al. (2014) for our four trees by assuming speciation rates to vary linearly as a function of time $\left(\mathbf{s}(\mathrm{t})=\mathbf{s}_{0}+\mathrm{rt}\right)$, where $\mathbf{s}_{0}$ is the speciation rate at present, $\mathrm{r}$ is the component associated with the variation in speciation rate through time and $t$ is the interval of time from the present to the past. As we were interested in the effect of time variation in speciation rates, dispersal and extinction rates were considered constant in the time-variable model. The results 
of the best-fit time-constant model were compared with the correspondent time-variable model using AIC scores to investigate the robustness of the observed patterns.

As the HiSSE method only deals with binary character states, we reclassified the widespread species as tropical or extratropical according to the predominant geographical range for each species. Since our GeoSSE models showed a higher diversification rate for tropical lineages (see results), we built, for each family, a character dependent model (BiSSE equivalent), and a character independent model in which we associated a "hidden" character state with the tropical state using the HiSSE notation. All models also included the percentage of species from each measured character state that were present in the phylogenies to deal with incomplete sampling. These models were compared using AIC scores to assess if the higher tropical diversification rates were indeed associated with the geographical character or if it is likely that other unmeasured character could account for the differences observed. We implemented these analyses in the R package 'hisse’ (Beaulieu \& O’Meara, 2016).

We assessed the Type I error rates associated with the GeoSSE model for our four families by building models with simulated neutral traits. By using the package 'diversitree', we simulated 100 phylogenies with initial parameters estimated from our totally constrained model ('evolutionary time') of the empirical GeoSSE model and with respective number of tips for each family. For each of these neutral trees, we estimated parameters from an unconstrained GeoSSE likelihood function and accessed the posterior distribution of estimated parameters using MCMC for 1000 generations. These distributions of likelihood ratios for each parameter were compared to check if there were differences among regions and the Type I error rates were calculated based on the proportion of neutral trees in which the estimated parameters differed among geographical regions. As suggested by Rabosky \& Goldberg (2015), we employed the results from the simulations to adjust the critical value of significance by dividing the threshold 
of 0.05 by the growth rate of spurious results (Type I error rates/0.05) for each parameter in the four families with the empirical dataset. For those parameters in which the Type I error rates were below the threshold of 0.05 , we kept this value as the critical significance level.

\section{RESULTS}

The families Chaetodontidae, Labridae and Pomacentridae contain predominantly tropical species, while the Sparidae has more extratropical than tropical species (Table 1). The set of best supported GeoSSE models showed that tropical lineages have higher speciation rates in all families (Fig. 2; Table 2). However, the results for extinction and dispersal rates varied among families.

For Chaetodontidae, the best supported model was the 'out of the tropics' model that prohibits between-region speciation (Table 2). Within this model, extinction rate was higher for extratropical lineages (Fig. 2A; Table 2). Dispersal rate was higher for tropical lineages expanding ranges into extratropical regions rather than the reverse (Fig. 2A; Table 2). Considering that speciation rate was higher in the tropics, we found that net diversification rate was negative for extratropical lineages (-0.446 lineages $\left.\mathrm{Myr}^{-1}\right)$ and positive for tropical lineages (0.132 lineages $\mathrm{Myr}^{-1}$; Fig. 2A).

The best-fit model for Labridae was also the 'out of the tropics' model prohibiting between-region speciation (Table 2). The estimate for extinction rate in this model was higher for extratropical lineages (Fig. 2B; Table 2), and the dispersal rate was found to be higher for tropical lineages expanding ranges into the extratropics (Fig. 2B; Table 2). Based on speciation and extinction estimates, we found positive diversification rates for both tropical $(0.123$ lineages $\mathrm{Myr}^{-1}$ ) and extratropical lineages (0.026 lineages $\mathrm{Myr}^{-1}$ ), although tropical net diversification was almost five times higher (Fig. 2B). 
For Pomacentridae the best-fit model was the 'evolutionary speed' model permitting between-region speciation (Fig. 2C; Table 2). The estimated value for the between-region speciation was similar to that estimated for extratropical speciation. Extinction rate was low for both tropical and extratropical lineages, causing the difference found for speciation rates to be the major influence in the observed difference in diversification rate among regions. We found a higher tropical net diversification rate (0.108 lineages Myr-1) compared with the extratropics (0.029 lineages Myr-1; Fig. 2C).

Within the Sparidae, the best supported model was also the 'out of the tropics' model, but permitting between-region speciation (Fig. 2D; Table 2). The estimated between-region speciation was lower than extratropical and tropical speciation rates in this model. Dispersal rate was higher for tropical lineages expanding ranges into the extratropics rather than in the opposite direction (Fig. 2D; Table 2). As in the Pomacentridae, the estimated extinction rate for Sparidae was low for both regions, which resulted in a higher tropical net diversification rate (0.062 lineages $\left.\mathrm{Myr}^{-1}\right)$ compared with the extratropics (0.033 lineages $\mathrm{Myr}^{-1}$; Fig. 2D).

Although the AIC results supported the 'out of the tropics' as the best model for Chaetodontidae, Labridae and Sparidae (Table 2), our simulations for model selection showed that given the available phylogenetic data, it is not possible to discriminate this model from the 'evolutionary speed' in all four families (Figure S5). Therefore, we can consider that these two models equally fit our data. These results do not change the overall picture since the speciation rates estimated under the 'evolutionary speed' model were still higher for tropical lineages in all families, however it limits our inferences about the roles of extinction and dispersal.

When we considered time variation in evolutionary rates, the models received little support compared to time-constant models in Pomacentridae and Sparidae (Table S2), indicating little variation in rates through time. For Chaetodontidae and Labridae, the time- 
variable model received support against the time-constant model, indicating that evolutionary rates varied through time in these families. However, the differences among tropical and extratropical rates showed the same pattern as in the time-constant models (Table S2), which highlights the robustness of the predicted differences among regions in the these models.

The comparison of character dependent (BiSSE equivalent) and character independent (HiSSE) models showed that for the Chaetodontidae, Pomacentridae and Sparidae, the presence of unmeasured characters could not account for rate differences, since the character dependent model received more support (Table S3). The pattern of higher tropical diversification rates was maintained in the character dependent model for these families, which is consistent with GeoSSE results. However, for Labridae, the character independent model received more support (Table S3), indicating that some unmeasured character might be driving the pattern of higher tropical diversification. When we plotted the net diversification along the labrid phylogeny, we detected higher rates associated with the tropical clade that includes the genus Thalassoma + Gomphosus and the tropical clade including the genus Scarus + Chlorurus (Fig. 3). These results does not invalidate the GeoSSE results as extratropical lineages were still associated with lower net diversification rates, however, they show that most tropical lineages had intermediate diversification rates, while two tropical clades displayed exceptionally higher net diversification (Fig. 3; Table S3).

The simulation results showed that the Type I error rates of the GeoSSE model are moderate to low. For speciation rates, the maximum error rates were 0.1 in Chaetodontidae, while for net diversification we detected an error rate of 0.11 in the Labridae (Table S4). For all other parameters, the GeoSSE model performed well in all families and we found Type I error rates around 0.06 and 0.09 with some remaining below the 0.05 threshold. By using these 
values to adjust the critical significance value for the empirical dataset, we found no differences in results of parameter comparisons among geographical regions (Fig. 2).

\section{DISCUSSION}

We found marked differences in tropical and extratropical evolutionary rates with tropical lineages showing higher diversification, mainly driven by more speciation in the tropics. In addition, we report a tendency of higher dispersal rates for tropical lineages expanding ranges into the extratropics. This suggests that the majority of extratropical lineages have arisen from tropical ones. These results support the predictions of the 'out of the tropics' model of evolution (Jablonski et al., 2006), although we also found support for the 'evolutionary speed' hypothesis (Rohde, 1992). Our results confirm the tropics as an important evolutionary engine for marine environments (Briggs, 2003), and highlight the complementarity of the predictions concerning the evolutionary dynamics behind latitudinal differences in biodiversity for reef fishes. Moreover, we find that two tropical Labridae lineages exhibit exceptionally higher diversification than that expected by latitudinal differences alone.

Most reef fishes depend on available coral reef habitats, so geological climatic changes that affected these environments might have also had a negative influence in their persistence (Cowman \& Bellwood, 2011; Pellissier et al., 2014). The areas in which coral reef habitats remained stable over geological times served as refugia from extinction during periods of drastic climatic fluctuations, such as the Pleistocene glacial cycles (Pellissier et al., 2014). Even though habitat loss resulting from climatic oscillations and tectonic activity are likely to have caused extinctions in tropical fish and other reef associated lineages in the past (Renema et al., 2008), fracturing and isolation of refugia may have also increased potential for speciation in some tropical regions (Pellissier et al., 2014). Our results show that this effect might be less 
pronounced for extratropical lineages, with the lack of fragmented or stable extratropical refugia resulting in lower rates of speciation. Peripheral extratropical reefs may have historically acted as reef fish biodiversity sinks. In particular for Chaetodontidae, the estimated negative net diversification indicates that the extratropics might be a recipient of lineages through speciation and dispersal from the tropics with little subsequent speciation to counteract the extratropical higher rate of extinction.

Patterns of diversification have previously been examined for several reef fish groups, with reef association linked to higher clade diversity (Alfaro et al., 2007; Cowman \& Bellwood, 2011). Here, we show that reef fish diversification patterns also follow a clear geographical trend of higher diversification rates for tropical lineages compared to extratropical ones. Our estimated values for tropical net diversification in Chaetodontidae, Labridae and Pomacentridae are very similar to whole family estimates calculated by Cowman \& Bellwood (2011), reflecting the predominance of tropical species within these families. As for Sparidae, our finding of a higher tropical lineage diversification is a surprising result considering that most of the contemporary species within this family are associated with extratropical environments. This suggests that the actual species richness distribution within this family might be greatly influenced by lineage dispersal. By comparing our results to other works that used a similar approach but different taxa (Pyron \& Wiens, 2013; Pyron, 2014; Rolland et al., 2014), it is possible to infer that higher tropical net diversification might be a general pattern for vertebrates across different phylogenetic scales and in both terrestrial and marine environments.

In addition to the geographical results, we show that exceptionally high rates of lineage diversification are associated with two predominantly tropical clades in the family Labridae. Similar rate shifts in the Labridae have been reported previously (Alfaro et al., 2009), associated with the tribe Julidines and a clade containing Scarus + Chlorurus. Here, we show that the rate 
shifts previously reported to be associated with the Julidines might represent a trickle-down effect driven by the sub-clade including Thalassoma + Gomphosus (Fig. 3). In the case of Scarus + Chlorurus, the previously identified shift in diversification rate was associated with their pronounced dichromatism and other social and behavioral characters related to sexual selection (Alfaro et al., 2009; Kazancioglu et al., 2009). It is possible that this same trait might also explain an elevated diversification rate in the clade Thalassoma + Gomphosus, since species within this group also display extreme sexual dichromatism (Kuiter, 2010). If true, then these two tropical clades might have undergone a sequential pattern of diversification (Streelman \& Danley, 2003) driven by the development of complex mating behaviors and phenotype throughout sexual selection. But, a number of other factors could also be involved. Both clades also have a complex longitudinal biogeographic history (Cowman \& Bellwood, 2013). Both clades have pantropical distributions (Choat et al., 2012; Bernardi et al., 2004), and in the case of Thalassoma, speciation and rapid range overlap have already been identified (Quenouille et al., 2011). Other functional traits such as diet have also been shown to play an 2013; Rabosky \& Goldberg, 2015), the differences in rate estimates we observe in the present study are consistent across families in the modeling procedure and robust when accounting for time variation. Moreover, our simulation results show that the Type I error rates from the GeoSSE model might be well below those found for the BiSSE model (Rabosky \& Goldberg, 2015), which reinforces the robustness of the differences found among geographical regions. With the Monte Carlo method, we also assessed the power of our analysis to detect real differences between models, which has been considered a major issue in phylogenetic 
comparative methods (Boettiger et al., 2012). The results from this model selection framework showed that both the 'out of the tropics' and the 'evolutionary speed' models equally fit our data and the direction of parameters estimated by these models were consistent with each evolutionary hypothesis. This highlights that differential rates of speciation have played a major and consistent role in the latitudinal differences in biodiversity for reef associated fishes, although we cannot discard extinction and dispersal as possible drivers of this pattern. generated and maintained reef fish diversity through time. Processes such as tectonic activity, changes in sea level, oceanographic conditions and geomorphological configuration are also recognized as important drivers of extant patterns of reef fish diversity (Renema et al., 2008; Bellwood et al., 2012). While these processes have been associated with the marked longitudinal diversity gradient presented by reef fishes and other reef associated organisms, their influence on the latitudinal gradient has yet to be fully determined. A better understanding of how these processes influenced the evolution of reef fishes by altering rates of speciation, extinction and dispersal is needed. This will allow us to disentangle the historical, biogeographic and environmental factors and how they have interacted to shape global reef fish diversity patterns.

\section{CONCLUSIONS}

Our study suggests that the tropics enhance lineage origination and may have reduced lineage extinction rates in reef fishes while being a fountain of evolutionary lineages to extratropical environments. We also highlight two tropical lineages in the family Labridae 
436 dichromatism. Alternatively, higher diversification in these clades might have been driven by

437 longitudinal biogeography across the marine tropics. Hence, our study proposes that speciation, 438 extinction and dispersal are key processes that generate and maintain higher tropical reef fish 439 species richness. To our knowledge, this is the first time that these processes have been 440 examined across multiple reef fish groups to reveal the mechanisms that promote latitudinal 441 differences in biodiversity. We suggest that further studies should explore how these 442 macroevolutionary dynamics influence other patterns in marine biodiversity.

\section{ACKNOWLEDGEMENTS}

We would like to thank B. Frédérich, F. Santini and S. Klanten for making their 446 phylogenies available. For comments and discussions on earlier drafts we also thank R.A. 447 Morais, E.L.H. Giehl, M.R. Pie, A. Lindner and three anonymous reviewers. This work was 448 financially supported by the Brazilian agencies CAPES (A.C.S. master's grant), CNPq (S.R.F. 449 grant - 309472/2011-3), FUNDECT (L.G.R.O.S. grant - 31445.448.13799.18082014), and by 450 the Yale Institute for Biospheric Studies - YIBS (P.F.C. Gaylord Donnelley Postdoctoral 451 Environmental Fellowship). 


\section{REFERENCES}

453

454

455

456

457

458

459

460

461

462

463

464

465

466

467

468

469

470

471

472

473

474

475

476

Alfaro, M.E., Brock, C.D., Banbury, B.L. \& Wainwright, P.C. (2009) Does evolutionary innovation in pharyngeal jaws lead to rapid lineage diversification in labrid fishes? $B M C$ evolutionary biology, 9, 255.

Alfaro, M.E., Santini, F. \& Brock, C.D. (2007) Do reefs drive diversification in marine teleosts? Evidence from the pufferfish and their allies (Order Tetraodontiformes). Evolution, 61, 2104-2126.

Allen, G.R. (1991) Damselfishes of the world, MERGUS Publishers, Melle.

Beaulieu, J.M. \& O’Meara, B.C. (2016) Detecting hidden diversification shifts in models of trait-dependent speciation and extinction. Systematic Biology, doi: 10.1093/sysbio/syw022.

Bellwood, D.R., Hughes, T.P., Connolly, S.R. \& Tanner, J. (2005) Environmental and geometric constraints on Indo-Pacific coral reef biodiversity. Ecology Letters, 8, 643651.

Bellwood, D.R., Renema, W. \& Rosen, B.R. (2012) Biodiversity hotspots, evolution and coral reef biogeography. Biotic Evolution and Environmental Change in Southeast Asia (ed. by D.J. Gower, K. Johnson, J. Richardson, B. Rosen, L. Rüber, and S. Williams), pp. 232. Cambridge University Press, Cambridge.

Bellwood, D.R. \& Wainwright, P.C. (2002) The history and biogeography of fishes on coral reefs. Coral reef fishes: dynamics and diversity on a complex ecosystem (ed. by P. Sale), pp. 5-32. Academic Press, San Diego.

Bernardi, G., Bucciarelli, G., Costagliola, D., Robertson, D.R. \& Heiser, J.B. (2004) Evolution of coral reef fish Thalassoma spp. (Labridae). 1. Molecular phylogeny and biogeography. Marine Biology, 144, 369-375.

Boettiger, C., Coop, G., Ralph, P. (2012) Is your phylogeny informative? Measuring the power of comparative methods. Evolution, 66, 2240-2251.

Briggs, J.C. (2003) Marine centres of origin as evolutionary engines. Journal of Biogeography, 30, 1-18.

Briggs, J.C. (1974) Marine zoogeography, McGraw-Hill, New York.

Choat, J.H., Klanten, O.S., Van Herwerden, L., Robertson, R.D. \& Clements, K.D. (2012) Patterns and processes in the evolutionary history of parrotfishes (Family Labridae). Biological Journal of the Linnean Society, 107, 529-557. 
Cowman, P.F. \& Bellwood, D.R. (2011) Coral reefs as drivers of cladogenesis: expanding coral reefs, cryptic extinction events, and the development of biodiversity hotspots. Journal of Evolutionary Biology, 24, 2543-2562.

Cowman, P.F. \& Bellwood, D.R. (2013) The historical biogeography of coral reef fishes: global patterns of origination and dispersal. Journal of Biogeography, 40, 209-224.

Davis, M.P., Midford, P.E. \& Maddison, W. (2013) Exploring power and parameter estimation of the BiSSE method for analyzing species diversification. BMC Evolutionary Biology, 13, 38.

Dowle, E.J., Morgan-Richards, M. \& Trewick, S.A. (2013) Molecular evolution and the latitudinal biodiversity gradient. Heredity, 110, 501-510.

Ebeling, A.W. \& Hixon, M.A. (1991) Tropical and temperate reef fishes: comparison of community structures. The Ecology of Fishes on Coral Reefs (ed. by P.F. Sale), pp. 509563. Academic Press, San Diego.

Eschmeyer, W.N. (2014) Catalog of Fishes. Version 2014.8, http://research.calacademy.org/research/ichthyology/catalog/fishcatmain.asp.

Fischer, A.G. (1960) Latitudinal variations in organic diversity. Evolution, 14, 64-81.

FitzJohn, R.G. (2012) Diversitree: comparative phylogenetic analyses of diversification in R. Methods in Ecology and Evolution, 3, 1084-1092.

Frédérich, B., Sorenson, L., Santini, F., Slater, G.J. \& Alfaro, M.E. (2013) Iterative ecological radiation and convergence during the evolutionary history of damselfishes (Pomacentridae). The American Naturalist, 181, 94-113.

Froese, R. \& Pauly, D. (2014) FishBase. Version 2014.8, www.fishbase.org.

Goldberg, E.E., Lancaster, L.T. \& Ree, R.H. (2011) Phylogenetic inference of reciprocal effects between geographic range evolution and diversification. Systematic Biology, 60, 451-465.

Hillebrand, H. (2004) On the generality of the latitudinal diversity gradient. The American Naturalist, 163, 192-211.

IUCN (2014) IUCN Red List of Threatened Species. Version 2014.2, www.iucnredlist.org.

Jablonski, D., Roy, K. \& Valentine, J.W. (2006) Out of the tropics: evolutionary dynamics of the latitudinal diversity gradient. Science, 314, 102-106.

Kazancioglu, E., Near, T.J., Hanel, R. \& Wainwright, P.C. (2009) Influence of sexual selection and feeding functional morphology on diversification rate of parrotfishes (Scaridae). Proceedings of the Royal Society B: Biological Sciences, 276, 3439-3446. 
Kuiter, R.H. (2010) Labridae fishes: Wrasses, Aquatic Photographics, Seaford.

Lobato, F.L., Barneche, D.R., Siqueira, A.C., Liedke, A.M.R., Lindner, A., Pie, M.R., Bellwood, D.R. \& Floeter, S.R. (2014) Diet and diversification in the evolution of coral reef fishes. PLOS ONE, 9, e102094.

Mittelbach, G.G., Schemske, D.W., Cornell, H. V, Allen, A.P., Brown, J.M., Bush, M.B., Harrison, S.P., Hurlbert, A.H., Knowlton, N., Lessios, H.A., McCain, C.M., McCune, A.R., McDade, L.A., McPeek, M.A., Near, T.J., Price, T.D., Ricklefs, R.E., Roy, K., Sax, D.F., Schluter, D., Sobel, J.M. \& Turelli, M. (2007) Evolution and the latitudinal diversity gradient: speciation, extinction and biogeography. Ecology Letters, 10, 315331.

Mora, C., Chittaro, P.M., Sale, P.F., Kritzer, J.P. \& Ludsin, S.A. (2003) Patterns and processes in reef fish diversity. Nature, 421, 933-936.

Paradis, E., Claude, J. \& Strimmer, K. (2004) APE: Analyses of Phylogenetics and Evolution in R language. Bioinformatics, 20, 289-290.

Parravicini, V., Kulbicki, M., Bellwood, D.R., Friedlander, A.M., Arias-Gonzalez, J.E., Chabanet, P., Floeter, S.R., Myers, R., Vigliola, L., D’Agata, S. \& Mouillot, D. (2013) Global patterns and predictors of tropical reef fish species richness. Ecography, 36, 1254-1262.

Pellissier, L., Leprieur, F., Parravicini, V., Cowman, P.F., Kulbicki, M., Litsios, G., Olsen, S.M., Wisz, M.S., Bellwood, D.R. \& Mouillot, D. (2014) Quaternary coral reef refugia preserved fish diversity. Science, 344, 1016-1019.

Pyron, R.A. (2014) Temperate extinction in squamate reptiles and the roots of latitudinal diversity gradients. Global Ecology and Biogeography, 23, 1126-1134.

Pyron, R.A. \& Wiens, J.J. (2013) Large-scale phylogenetic analyses reveal the causes of high tropical amphibian diversity. Proceedings of the Royal Society B: Biological Sciences, 280, 20131622.

Quenouille, B., Hubert, N., Bermingham, E. \& Planes, S. (2011) Speciation in tropical seas: allopatry followed by range change. Molecular Phylogenetics and Evolution, 58, 546552.

R Core Team (2014) R: A Language and Environment for Statistical Computing. $R$ Foundation for Statistical Computing, http://www.r-project.org.

Rabosky, D.L. \& Goldberg, E.E. (2015) Model inadequacy and mistaken inferences of traitdependent speciation. Systematic Biology, 64, 340-355.

Randall, J.E. (2005) Reef and shore fishes of the South Pacific, University of Hawaii Press, Honolulu. 
Renema, W., Bellwood, D.R. \& Braga, J.C. (2008) Hopping hotspots: global shifts in marine biodiversity. Science, 321, 654-657.

Ricklefs, R.E. (2006) Evolutionary diversification and the origin of the diversity-environment relationship. Ecology, 87, S3-13.

Rohde, K. (1992) Latitudinal gradients in species diversity: the search for the primary cause. Oikos, 65, 514-527.

Rolland, J., Condamine, F.L., Jiguet, F. \& Morlon, H. (2014) Faster speciation and reduced extinction in the tropics contribute to the Mammalian latitudinal diversity gradient. PLoS Biology, 12, e1001775.

Santini, F., Carnevale, G. \& Sorenson, L. (2014) First multi-locus timetree of seabreams and porgies (Percomorpha: Sparidae). Italian Journal of Zoology, 81, 55-71.

Streelman, J.T. \& Danley, P.D. (2003) The stages of vertebrate evolutionary radiation. Trends in Ecology and Evolution, 18, 126-131.

Tittensor, D.P., Mora, C., Jetz, W., Lotze, H.K., Ricard, D., Berghe, E. Vanden \& Worm, B. (2010) Global patterns and predictors of marine biodiversity across taxa. Nature, $\mathbf{4 6 6}$, 1098-1101.

Wiens, J.J. \& Donoghue, M.J. (2004) Evolutionary diversification and the origin of the diversity-environment relationship. Trends in Ecology \& Evolution, 19, 639-644.

\section{BIOSKETCH}

A.C. Siqueira obtained his MSc at the Universidade Federal de Santa Catarina, being supervised by S.R. Floeter in the Marine Macroecology and Biogeography Lab. His main research interests lies in historical, evolutionary and ecological processes that determine contemporary patterns in species distributions. 
578 TABLES

579 Table 1. Absolute number of species represented in phylogenies and the respective proportion in relation

580 to all recognized species by family in each character state: widespread (Wide), tropical (Trop),

581 extratropical (Extra).

\begin{tabular}{llll|lll|llll|lll}
\hline Family & \multicolumn{3}{c}{ Chaetodontidae } & \multicolumn{3}{c}{ Labridae } & \multicolumn{3}{c}{ Pomacentridae } & \multicolumn{3}{c}{ Sparidae } \\
\hline State & Wide & Trop & Extra & Wide & Trop & Extra & Wide & Trop & Extra & Wide & Trop & Extra \\
\hline Species & 32 & 58 & 5 & 80 & 169 & 54 & 25 & 174 & 7 & 33 & 24 & 34 \\
\% in Phylo & 0.89 & 0.71 & 0.56 & 0.62 & 0.44 & 0.67 & 0.57 & 0.56 & 0.33 & 0.83 & 0.59 & 0.79 \\
\hline
\end{tabular}

582

583 
$585(\triangle \mathrm{AIC})$, with respective degrees of freedom (d.f.), $\log$-likelihood $(\ln L)$ and Akaike Information Criterion

586 (AIC). Parameter estimates are: tropical speciation $\left(\mathbf{s}_{\mathrm{Trop}}\right)$, extratropical speciation ( $\left.\mathbf{s}_{\mathrm{Ex}}\right)$, between regions

587 speciation $\left(\mathbf{s}_{\mathrm{Btw}}\right)$, tropical extinction $\left(\mathbf{x}_{\text {Trop }}\right)$, extratropical extinction $\left(\mathbf{x}_{\mathrm{Ex}}\right)$, dispersal from the tropics

$588\left(\mathbf{d}_{\text {Trop }}\right)$, dispersal from the extratropics $\left(\mathbf{d}_{\mathrm{Ex}}\right)$.

\begin{tabular}{|c|c|c|c|c|c|c|c|c|c|c|c|}
\hline Models & d.f. & $\ln L$ & AIC & $\Delta \mathrm{AIC}$ & $\mathbf{S}_{\text {Trop }}$ & $\mathbf{S}_{\mathrm{Ex}}$ & $\mathbf{s}_{\mathrm{Btw}}$ & $\mathbf{x}_{\text {Trop }}$ & $\mathbf{x}_{\mathrm{Ex}}$ & $\mathbf{d}_{\text {Trop }}$ & $\mathbf{d}_{\mathrm{Ex}}$ \\
\hline \multicolumn{12}{|l|}{ Chaetodontidae } \\
\hline Out of the tropics $\left(\mathbf{s}_{\mathrm{Btw}}=0\right)$ & 6 & -332.9 & 677.9 & 0 & $2.7 \mathrm{e}-01$ & $3.6 \mathrm{e}-02$ & - & $1.4 \mathrm{e}-01$ & $4.8 \mathrm{e}-01$ & $3.2 \mathrm{e}-01$ & 3.9 e- 08 \\
\hline Out of the tropics & 7 & -332.9 & 679.9 & 2.0 & $2.7 \mathrm{e}-01$ & $3.5 \mathrm{e}-02$ & $2.4 \mathrm{e}-10$ & $1.4 \mathrm{e}-01$ & $4.8 \mathrm{e}-01$ & $3.2 \mathrm{e}-01$ & $2.3 \mathrm{e}-08$ \\
\hline Evolutionary speed $\left(\mathbf{s}_{\mathrm{Btw}}=0\right)$ & 4 & -336.0 & 680.1 & 2.2 & $2.8 \mathrm{e}-01$ & $3.5 \mathrm{e}-02$ & - & $1.5 \mathrm{e}-01$ & - & $1.5 \mathrm{e}-01$ & - \\
\hline Evolutionary speed & 5 & -336.0 & 682.1 & 4.2 & $2.8 \mathrm{e}-01$ & $3.5 \mathrm{e}-02$ & $1.5 \mathrm{e}-08$ & $1.5 \mathrm{e}-01$ & - & $1.5 \mathrm{e}-01$ & - \\
\hline Niche conservatism & 5 & -336.3 & 682.7 & 4.8 & $1.2 \mathrm{e}-01$ & - & $5.3 \mathrm{e}-01$ & $2.9 \mathrm{e}-01$ & - & $1.7 \mathrm{e}-01$ & 3.0 \\
\hline Niche conservatism $\left(\mathbf{s}_{\mathrm{Btw}}=0\right)$ & 4 & -337.4 & 682.9 & 5.0 & $2.4 \mathrm{e}-01$ & - & - & $2.9 \mathrm{e}-01$ & - & $1.0 \mathrm{e}-01$ & 1.6 \\
\hline Evolutionary time $\left(\mathbf{s}_{\mathrm{Btw}}=0\right)$ & 3 & -347.1 & 700.3 & 22.4 & $1.9 \mathrm{e}-01$ & - & - & $7.0 \mathrm{e}-02$ & - & 9.5 e- 02 & - \\
\hline Evolutionary time & 4 & -347.1 & 702.3 & 24.4 & $1.9 \mathrm{e}-01$ & - & 8.4 e- 09 & $7.0 \mathrm{e}-02$ & - & $9.5 \mathrm{e}-02$ & - \\
\hline \multicolumn{12}{|l|}{ Labridae } \\
\hline Out of the tropics $\left(\mathbf{s}_{\mathrm{Btw}}=0\right)$ & 6 & -1279.6 & 2571.2 & 0 & $1.2 \mathrm{e}-01$ & $6.1 \mathrm{e}-02$ & - & $5.6 \mathrm{e}-07$ & $3.5 \mathrm{e}-02$ & 4.9 e- 02 & $8.2 \mathrm{e}-03$ \\
\hline Out of the tropics & 7 & -1279.6 & 2573.4 & 2.2 & $1.2 \mathrm{e}-01$ & 5.9 e- 02 & $3.3 \mathrm{e}-06$ & $3.8 \mathrm{e}-07$ & $4.2 \mathrm{e}-02$ & $5.2 \mathrm{e}-02$ & $4.5 \mathrm{e}-06$ \\
\hline Evolutionary speed $\left(\mathbf{s}_{\mathrm{Btw}}=0\right)$ & 4 & -1284.7 & 2577.4 & 6.2 & $1.3 \mathrm{e}-01$ & $2.3 \mathrm{e}-02$ & - & $7.1 \mathrm{e}-03$ & - & $3.3 \mathrm{e}-02$ & - \\
\hline Evolutionary speed & 5 & -1284.7 & 2579.4 & 8.2 & $1.3 \mathrm{e}-01$ & $5.3 \mathrm{e}-02$ & $6.3 \mathrm{e}-09$ & $7.1 \mathrm{e}-03$ & - & $3.3 \mathrm{e}-02$ & - \\
\hline Evolutionary time $\left(\mathbf{s}_{\mathrm{Btw}}=0\right)$ & 3 & -1306.5 & 2619.0 & 48.2 & $1.0 \mathrm{e}-01$ & - & - & $9.2 \mathrm{e}-03$ & - & $3.0 \mathrm{e}-02$ & - \\
\hline Niche conservatism $\left(\mathbf{s}_{\mathrm{Btw}}=0\right)$ & 4 & -1305.7 & 2619.4 & 48.6 & $1.0 \mathrm{e}-01$ & - & - & $8.1 \mathrm{e}-03$ & - & $3.3 \mathrm{e}-02$ & $7.4 \mathrm{e}-07$ \\
\hline Evolutionary time & 4 & -1306.5 & 2621.0 & 50.2 & $1.0 \mathrm{e}-01$ & - & $8.1 \mathrm{e}-07$ & $9.1 \mathrm{e}-03$ & - & $3.0 \mathrm{e}-02$ & - \\
\hline Niche conservatism & 5 & -1305.7 & 2621.4 & 50.6 & $1.0 \mathrm{e}-01$ & - & $1.2 \mathrm{e}-06$ & $8.2 \mathrm{e}-03$ & - & $3.3 \mathrm{e}-02$ & $6.2 \mathrm{e}-06$ \\
\hline \multicolumn{12}{|l|}{ Pomacentridae } \\
\hline Evolutionary speed & 5 & -813.7 & 1637.5 & 0 & $1.0 \mathrm{e}-01$ & $2.9 \mathrm{e}-02$ & $1.7 \mathrm{e}-02$ & $5.8 \mathrm{e}-09$ & - & $1.5 \mathrm{e}-02$ & - \\
\hline Evolutionary speed $\left(\mathbf{s}_{\mathrm{Btw}}=0\right)$ & 4 & -815.7 & 1639.5 & 2 & $1.1 \mathrm{e}-01$ & $3.7 \mathrm{e}-02$ & - & 8.9 e- 03 & - & $1.5 \mathrm{e}-02$ & - \\
\hline Out of the tropics & 7 & -813.6 & 1641.2 & 3.7 & $1.0 \mathrm{e}-01$ & $3.6 \mathrm{e}-02$ & $1.7 \mathrm{e}-02$ & $5.2 \mathrm{e}-10$ & $2.0 \mathrm{e}-02$ & $1.8 \mathrm{e}-02$ & $1.0 \mathrm{e}-02$ \\
\hline Out of the tropics $\left(\mathbf{s}_{\mathrm{Btw}}=0\right)$ & 6 & -815.0 & 1642.1 & 4.6 & $1.0 \mathrm{e}-01$ & $5.5 \mathrm{e}-02$ & - & $3.6 \mathrm{e}-07$ & $4.9 \mathrm{e}-02$ & $2.1 \mathrm{e}-02$ & $7.9 \mathrm{e}-03$ \\
\hline Niche conservatism $\left(\mathbf{s}_{\mathrm{Btw}}=0\right)$ & 4 & -821.1 & 1650.2 & 12.7 & $1.0 \mathrm{e}-01$ & - & - & $9.4 \mathrm{e}-03$ & - & $1.1 \mathrm{e}-02$ & 6.6 e- 02 \\
\hline Niche conservatism & 5 & -820.1 & 1650.3 & 12.8 & $1.0 \mathrm{e}-01$ & - & $1.7 \mathrm{e}-02$ & $3.9 \mathrm{e}-03$ & - & $1.1 \mathrm{e}-02$ & $7.7 \mathrm{e}-02$ \\
\hline Evolutionary time $\left(\mathbf{s}_{\mathrm{Btw}}=0\right)$ & 3 & -822.3 & 1650.7 & 13.2 & $1.0 \mathrm{e}-01$ & - & - & $4.8 \mathrm{e}-03$ & - & $1.3 \mathrm{e}-02$ & - \\
\hline Evolutionary time & 4 & -821.6 & 1651.3 & 13.8 & $1.0 \mathrm{e}-01$ & - & $1.0 \mathrm{e}-02$ & $1.8 \mathrm{e}-03$ & - & $1.3 \mathrm{e}-02$ & - \\
\hline \multicolumn{12}{|l|}{ Sparidae } \\
\hline Out of the tropics & 7 & -429.3 & 872.7 & 0 & $6.2 \mathrm{e}-02$ & $3.3 \mathrm{e}-02$ & $1.6 \mathrm{e}-02$ & $2.3 \mathrm{e}-07$ & $2.4 \mathrm{e}-07$ & $6.7 \mathrm{e}-02$ & $7.1 \mathrm{e}-03$ \\
\hline Evolutionary speed & 5 & -433.5 & 877.0 & 4.3 & $1.6 \mathrm{e}-02$ & $2.8 \mathrm{e}-07$ & $2.2 \mathrm{e}-01$ & $2.8 \mathrm{e}-03$ & - & $1.2 \mathrm{e}-01$ & - \\
\hline Out of the tropics $\left(\mathbf{s}_{\mathrm{Btw}}=0\right)$ & 6 & -432.5 & 877.1 & 4.4 & $6.6 \mathrm{e}-02$ & $4.2 \mathrm{e}-02$ & - & $4.5 \mathrm{e}-03$ & $1.4 \mathrm{e}-02$ & 7.6 e- 02 & 5.4 e- 03 \\
\hline Evolutionary time $\left(\mathbf{s}_{\mathrm{Btw}}=0\right)$ & 4 & -435.3 & 878.6 & 5.9 & 8.4 e- 03 & - & $2.2 \mathrm{e}-01$ & $4.1 \mathrm{e}-03$ & - & $1.2 \mathrm{e}-01$ & - \\
\hline Niche conservatism $\left(\mathbf{s}_{\mathrm{Btw}}=0\right)$ & 4 & -435.4 & 878.8 & 6.1 & $5.4 \mathrm{e}-02$ & - & - & $8.1 \mathrm{e}-03$ & - & $6.0 \mathrm{e}-02$ & $1.9 \mathrm{e}-02$ \\
\hline Evolutionary time & 3 & -436.8 & 879.7 & 7.0 & $5.5 \mathrm{e}-02$ & - & - & $1.0 \mathrm{e}-02$ & - & $4.0 \mathrm{e}-02$ & - \\
\hline Niche conservatism & 5 & -435.2 & 880.4 & 7.7 & 8.8 e- -03 & - & $2.1 \mathrm{e}-01$ & $3.6 \mathrm{e}-03$ & - & $1.3 \mathrm{e}-01$ & $1.1 \mathrm{e}-01$ \\
\hline Evolutionary speed $\left(\mathbf{s}_{\mathrm{Btw}}=0\right)$ & 4 & -436.6 & 881.3 & 8.6 & $6.0 \mathrm{e}-02$ & $5.2 \mathrm{e}-02$ & - & $9.8 \mathrm{e}-03$ & - & 3.9 e- 02 & - \\
\hline
\end{tabular}




\section{FIGURE LEGENDS}

593 Figure 1. Map showing tropical (red) and extratropical (blue) reef locations around the world defined 594 by the isocrym of $20^{\circ} \mathrm{C}$, which is considered the latitudinal distribution limit for tropical marine fauna 595 (Briggs, 1974).

596

Figure 2. Rates of speciation, extinction and net diversification (speciation minus extinction) for tropical (red) and extratropical (blue) lineages of four reef fish families. Dispersal rates are also shown with tropical lineages expanding ranges into extratropical environments (green) and extratropical lineages expanding ranges into the tropics (yellow). Probability density plots are based on 1000 Markov Chain Monte Carlo samples of the best-fit model for each family under GeoSSE. Vertical lines represent parameter estimates based on Maximum Likelihood of the best-fit GeoSSE model for each family. Credibility intervals were adjusted by the Type I error rates calculated for each parameter using the results from GeoSSE simulations in each family. For Pomacentridae and Sparidae the best-fit model permitted the between regions speciation parameter (purple) to be $>0$. For Pomacentridae, dispersal and extinction were found to be equal for both tropical and extratropical lineages (shown in grey).

Figure 3. Labridae phylogeny built under HiSSE notation, showing tropical lineages (black branches) and extratropical lineages (white branches), with respective net diversification rates (color gradient from blue - lower diversification - to red - higher diversification). Four clades are highlighted, being two tropical clades with high net diversification rates (Scarus + Chlorurus and Thalassoma + Gomphosus), and two extratropical clades with low net diversification rates (Labrines and Odacines). The graph shows the frequency of net diversification rates on the tips of the phylogeny, highlighting that there are few lineages with high rates and more lineages with lower to intermediate rates.

615 
617 FIGURES

$618 \quad$ Figure 1.

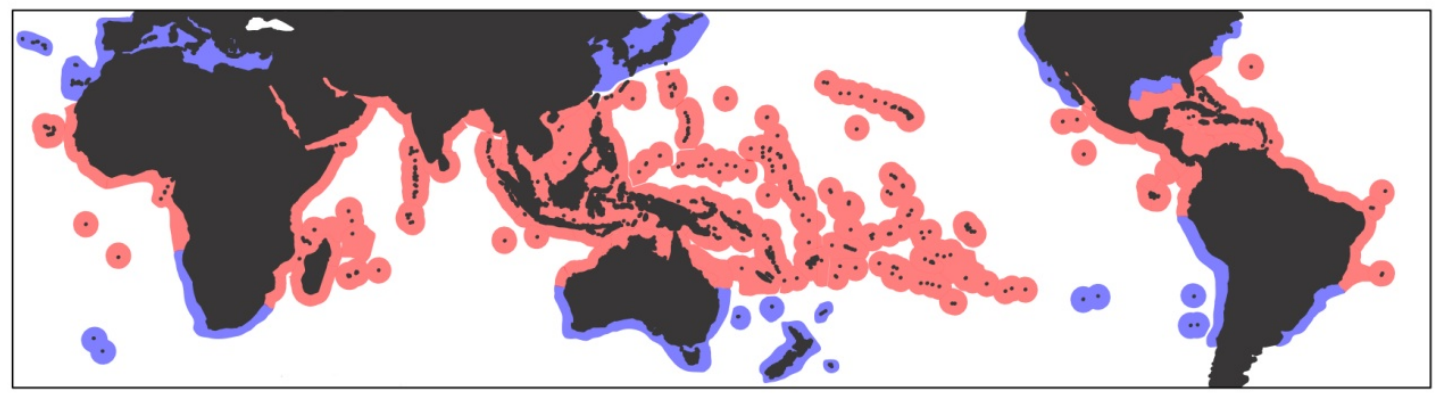

619

620 
Figure 2.

A)

$$
\begin{aligned}
& \text { Speciation } \\
& \text { Tropical } \\
& \text { Extratropical } \\
& \text { Between }
\end{aligned}
$$

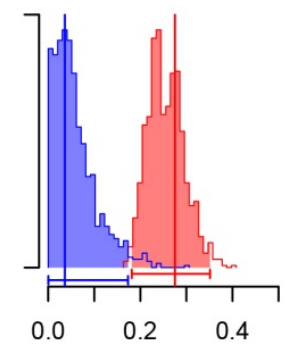

B)
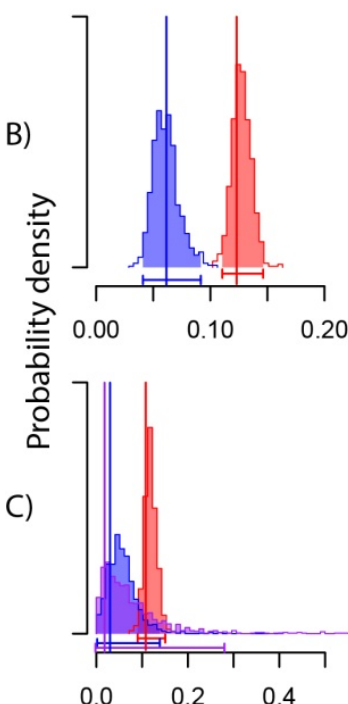

D)

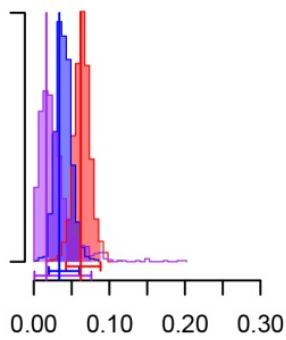

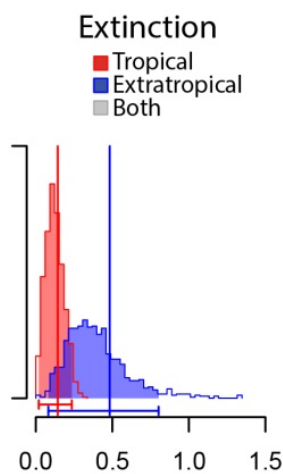

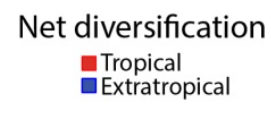

\section{Dispersal}

$\square$ Tropical-->Extratropical $\square$ Extratropical-->Tropical
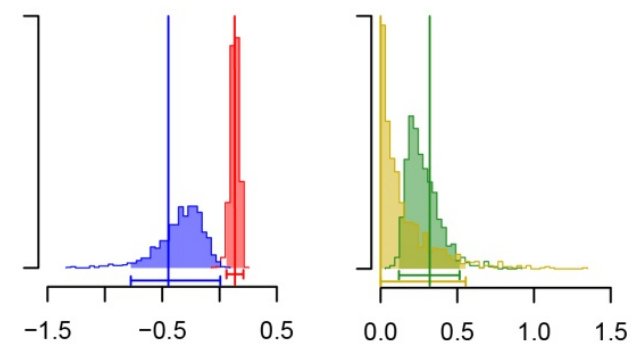

Chaetodontidae
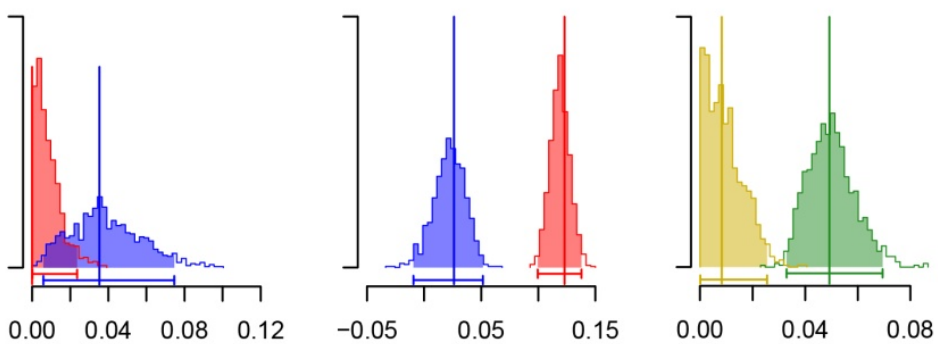

Labridae
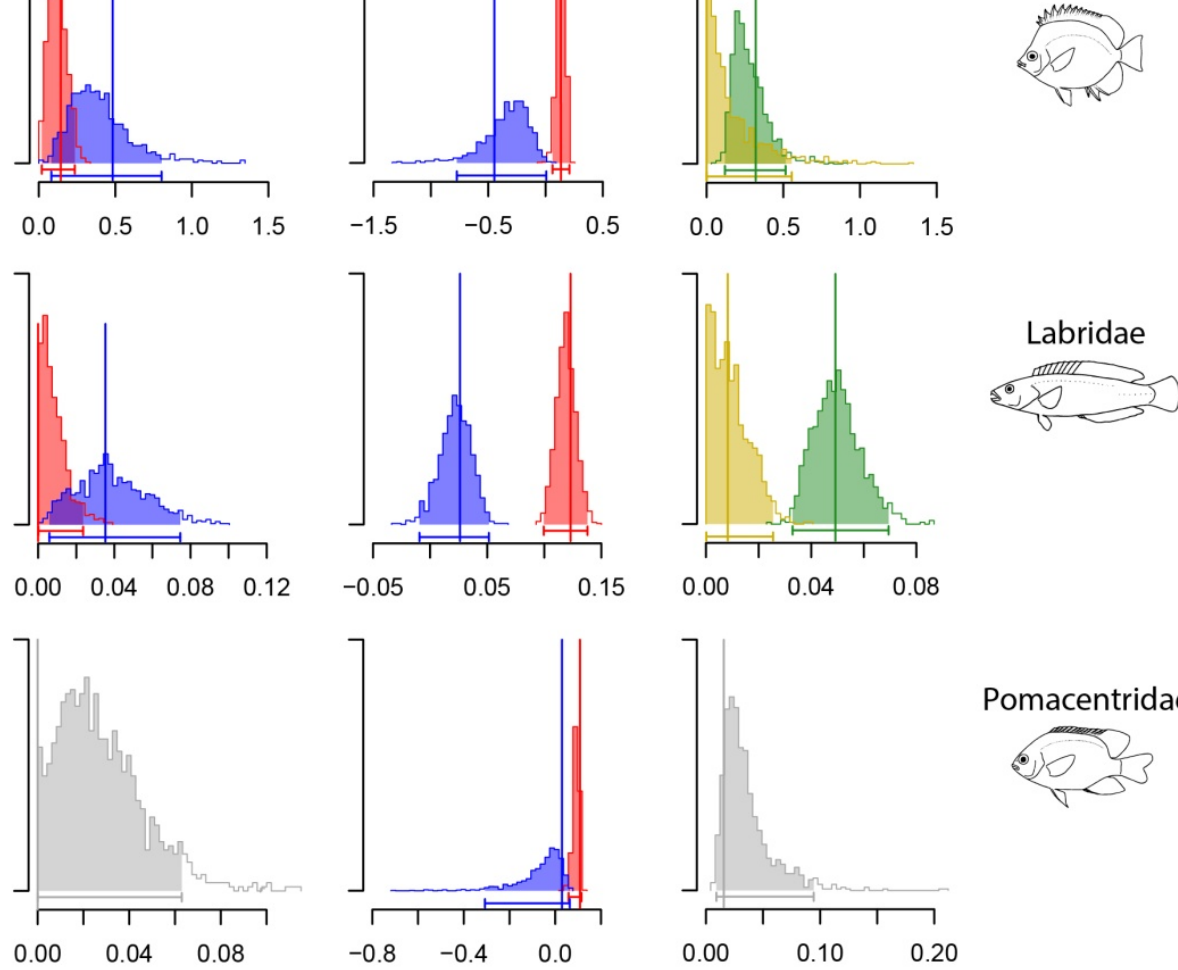

Pomacentridae
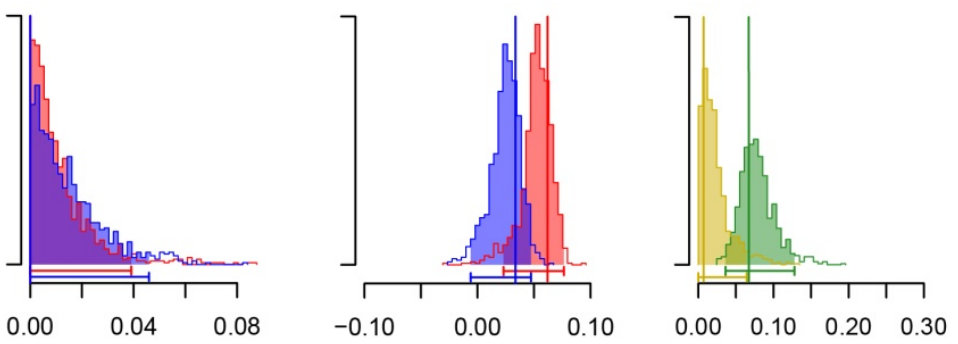

Sparidae

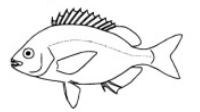

Parameter estimate 
Figure 3.

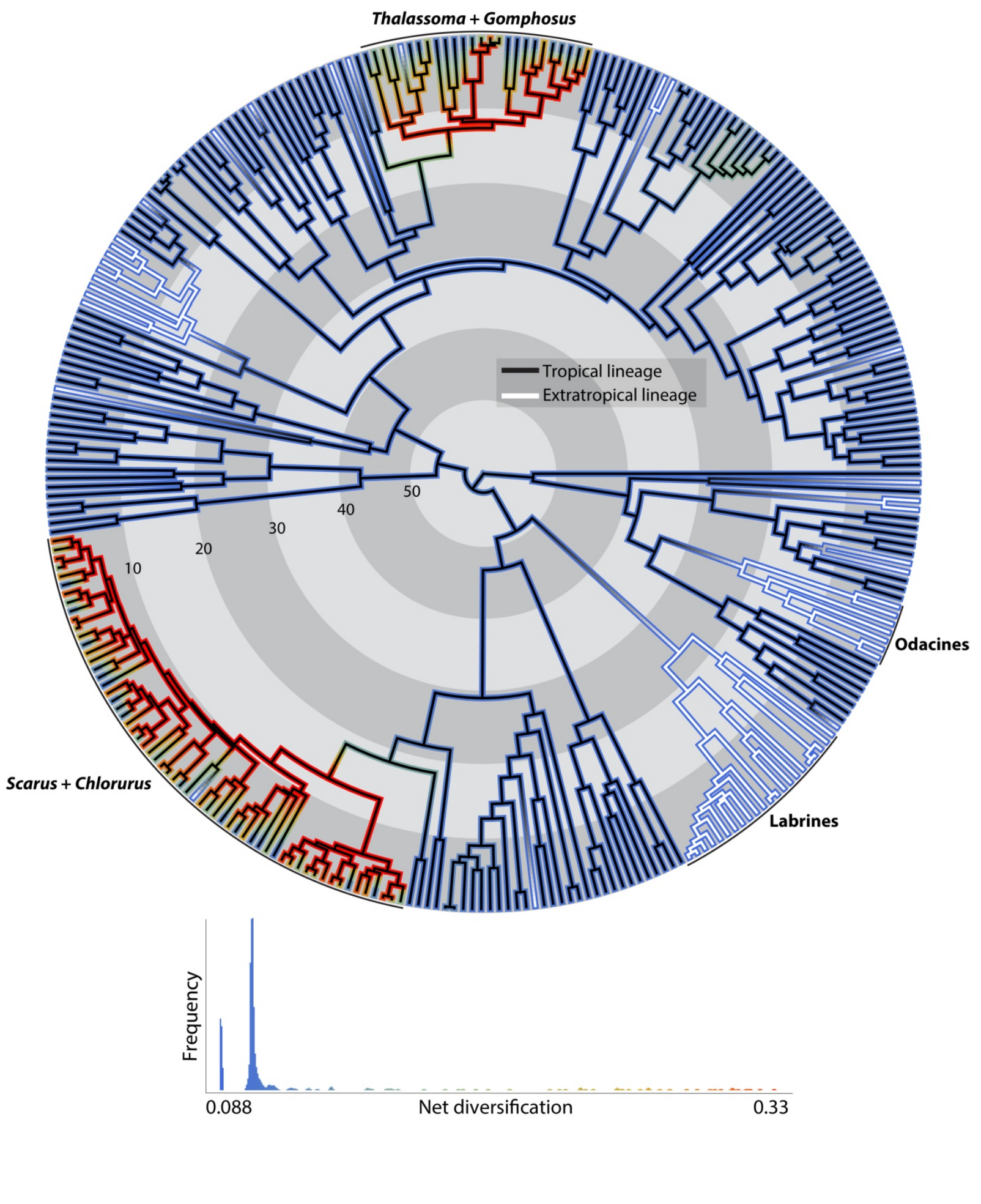

